\title{
Investigating the Potential of In-Home Devices for Improving Medication Adherence
}

\author{
Young S. Lee, Joe Tullio, Nitya Narasimhan, Pallavi Kaushik, Jonathan R. Engelsma, and Santosh Basapur \\ Applied Research and Technology Center \\ Motorola, Inc. \\ Schaumburg, IL USA \\ \{youngLee, joe.tullio, nitya, pkaushik, Jonathan.Engelsma, sbasapur\}@motorola.com
}

\begin{abstract}
We conducted five focus groups with seniors and middle-aged participants who live independently in their own homes to assess the potential value of a home-centered medication reminder system concept. The medication reminder system was conceptualized as a system that uses a television and set-top box, mobile phones and other in-home accessories as a means to set and deliver medication reminders. We found that the main value perceived by participants in the medication reminder system was its ability to provide multiple channels for them to be reminded of medications. The mobile phone, due to its advantages in portability and privacy, was considered to be the most useful device on which to receive reminders. Most participants saw value in receiving secondary reminders on other devices in their home such as the TV, PC, and other in-home accessories. Design implications along with other findings about the challenges faced by participants in managing their medications are discussed.
\end{abstract}

Keywords-component; medication reminder system; user needs; medication management; medication adherence;

\section{INTRODUCTION}

Poor medication adherence still remains a major challenge facing most industrialized countries including the United States, leading to worsening disease severity and increased costs associated with higher hospital admission rates [1]. According to the American Heart Association, more than half of all Americans with chronic disease do not follow their physician's medication and lifestyle guidance, and nine out of ten make mistakes taking their medication [2]. In the U.S. alone, non-adherence to medications causes 125,000 deaths annually and accounts for $10 \%$ to $25 \%$ of hospital and nursing home admissions, with the annual direct and indirect cost of non-adherence estimated to be over $\$ 177$ billion [3]. Recent literature show that, despite extensive research into interventions for assisting with adherence, rates of adherence have not changed over the past three decades [4,5]

Previous research found that forgetfulness is one of the most common factors contributing to poor adherence, along with the complexity of the regimen and disruption of daily routines $[6,7]$. Other studies have shown that medications are taken at various locations and in various contexts within the home; therefore, home computers are of marginal utility in this space since few people take their medications near them [8,9]. All of these results suggest that automated medication reminders through multiple devices within the home might be helpful technology interventions for improving adherence.

As part of an initiative investigating technology solutions to support in-home health care activities, we created several concepts of a multi-device, home-centered system that would use television (TV) along with set-top box (STB), mobile phones and other in-home devices as a means to set and deliver medication reminders. To assess the value of these concepts, we conducted a focus group study with the following goals: 1) to understand the current practices and challenges faced by our potential users (i.e., middle aged and senior adults living independently) in managing their medications, 2) to assess the potential value of TV (\& STB), mobile phones and other inhome devices for delivering medication reminders, and 3) to identify additional user needs to support home-centered medication management activities. In this paper, we review previous research on medication adherence and medication reminders, describe our study design, and present the results. We conclude with a discussion of design implications derived from these results, along with supporting evidence found in the literature.

\section{RELATED WORK}

There has been extensive research to identify the factors that contribute to poor adherence, and to develop technological interventions for improving adherence. Ownby [6] found that poor medication adherence in older adults is a complex phenomenon that depends on a combination of medical, medication, personal, and economic factors. He outlined eight key risk factors for non-adherence: 1) low levels of health literacy, 2) poor understanding of the purpose for medication, 3) poor understanding of the impact of a medication on health outcomes, 4) memory or general cognitive impairment, 5) living alone or not having a caregiver, 6) regimen complexity, 7) communication difficulties between physician and patient, 8) lack of insurance or other inability to pay for medication. Similarly, Osterberg and Blaschke [7] reported that barriers to adherence are created from each of the three players in the healthcare: patient (e.g., forgetfulness, other priorities, decision to omit does, lack of information and emotional factors), 
physicians (e.g., prescribing complex regimens, failing to explain the benefits and side effects of medications, not giving consideration to the patients' lifestyle), and health care systems (e.g., limiting access to health care and having high costs for drugs, copayments). All of these studies suggest that medication adherence is a complex phenomenon with multiple determining factors, and that the use of technology-based interventions can be a useful strategy for improving adherence. For example, the creation and delivery of individually-tailored educational materials can be automated through a computerbased application. Other automated interventions like computer-based reminders can also improve medication adherence [6].

Recently, Palen and Aaløkke [10] conducted an extensive ethnographic study investigating the medication management practices of elders with the objective of informing the design of in-home assistive technology to support medication adherence. This study identified a number of practices that employed a kind of "socially distributed cognition" in order to manage the organization and consumption of medications. Pills were often placed near locations associated with daily routines, and distinctions were made between important prescriptions and those that were seen as less critical, such as over-the-counter pills and supplements. From this analysis, they suggested that assistive technology needs to be flexible to support personalized medication reminding while being distributed across the home using spatial arrangements in places that support routines. Subsequent work by Balegaard et al. [11] outlined the need for integrating technology with as little disruption as possible, and stressed that users must understand the purpose of the technology that is given to them.

\section{METHOD}

\section{A. Concepts and study design}

We created four scenarios to illustrate the concepts of the multi-device medication reminder system. We briefly describe each scenario below:

- Scenario 1: A hypothetical user (John) receives a reminder on his television indicating how many medications are due to be taken now. He was able to set this reminder earlier using his television remote control.

- Scenario 2: Here, the same reminder is due, but John is away from the house and therefore receives the reminder on his mobile phone. Simultaneously, his spouse (Caryn), who is at home, sees the same reminder appear on the television. She chooses to call John to remind him as well.

- Scenario 3: Caryn and John receive a reminder from their digital picture frame while cooking dinner at home. John snoozes it for an hour. He later receives a reminder on his television while watching TV after dinner.

- Scenario 4: John receives a phone call from his daughter whom he elected to have notified in case he missed his medications. In addition, John receives an email from his doctor because he has elected to share his medication intake with his doctor. The doctor can call or email him if the regimen changes or isn't being followed properly.

Note that while the earlier scenarios serve to examine the basic premise of using in-home devices as reminder displays, the latter scenarios are designed to push the issues of privacy, autonomy, and ubiquity in terms of how medication information is shared and communicated.

\section{B. Participants}

We recruited a total of 28 participants to participate in five focus groups. We used a third-party service to recruit participants with the following characteristics:

- Experience with technology (TV, mobile phone, internet/email)

- Take at least 6 different medications on a regular schedule

- Live independently

- Perceive a need for assistance in taking their medication.

Table 1 shows the breakdown of the age and gender for participants in each group.

TABLE I. PARTICIPANT GROUP DEMOGRAPHICS

\begin{tabular}{|l|l|l|}
\hline Group & Age range & Gender \\
\hline 1 & $40-55$ & 6 Male \\
\hline 2 & $56-70$ & 6 Male \\
\hline 3 & $40-55$ & 5 Female \\
\hline 4 & $56-70$ & 5 Female \\
\hline 5 & Over 70 & 4 Female, 2 Male \\
\hline
\end{tabular}

As per the criteria described above, all participants were frequent TV users $($ Mean $=4 \mathrm{hrs} /$ day; $M i n=3 ; M a x=10)$, took an average of 8.6 medications regularly $(M i n=4 ; M a x=16)$, and indicated some need for medication reminders. The need for medication reminders was measured in two questions - implicit in forgetfulness (Q: "Sometimes I forget to take medications") and explicit in a desire for reminders (Q: "I need reminders to help me take my medications on time"). A four-point Likerttype scale $(0=$ Strongly disagree; $4=$ Strongly agree $)$ was used to measure these two attributes.

\section{Apparatus and procedure}

We began the focus group by asking about the specific difficulties our participants have in terms of managing their medications and the strategies they employ to cope with these challenges, if any. Next, we presented our medication reminder system concepts (see Figure 1 for an example) using 
storyboards that represent the concepts described earlier, followed by open discussions about the potential value and specific concerns in using the system. Each focus group lasted about 2.5 hours. Participants were compensated with a $\$ 100$ gift check. Focus groups were videotaped and later transcribed for data analysis.

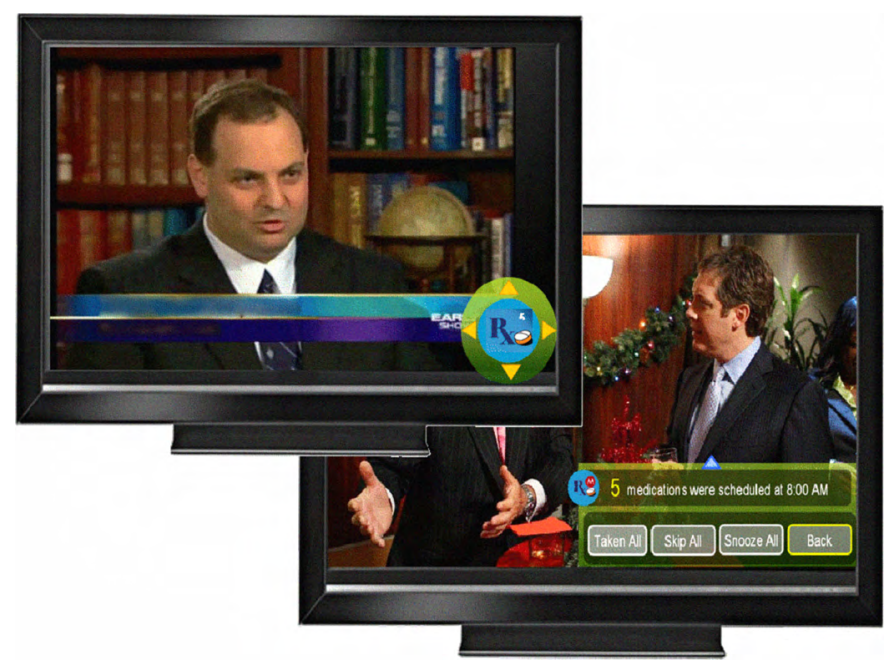

Figure 1. TV reminder concepts used in our focus groups, with a basic reminder (top left), and additional details (bottom right).

\section{Data Analysis}

An interpretive group-based analysis was conducted, following Thompson et al. [12]. We formed an interpretive group with four researchers. The analysts first individually reviewed the focus group videos and transcribed them. Upon the completion of the transcription, the analysts worked together to develop structural open codes based on questions asked by a moderator. Next, each analyst conducted a thematic analysis for the assigned portion, guided by the initial set of focus questions for analysis. In the thematic analysis, we identified items for analysis, focusing on statements and implications, and we then put these items together into groups or categories. Upon the completion of individual analysis, the interpretive group shared the resulting codes and the procedure with other analysts for verification as a way to ensure qualitative rigor [13]. Finally, the detailed process of "comparison, contrast, and integration" was conducted to identify patterns or themes that were not part of our research focus as an exploration step [13]. Through the entire analysis, we used a qualitative analysis tool, MAXQDA [14].

\section{RESULTS}

\section{A. Challenges for medication management}

We identified a number of difficulties cited by our participants in taking medications. From their perspective, problems arise in both the consumption of medications and the logistics of medications. We describe these challenges below.
1) Forgetfulness: Participants expressed a wide range of challenges in terms of keeping up with their medication regimens. Among the various challenges, forgetfulness was an oft-cited problem, but it was due to a number of different reasons, such as fatigue or distraction at the time of medication intake. Participants cited being too groggy, busy, or occupied with their children in the morning to remember their medications, but others also mentioned being too fatigued at night to remember before falling asleep. For example, participant P1.5 stated, "I mostly forget at night time when I get back from work...and I'm tired from a long day....and fall asleep and I miss taking it." In addition, distractions played a large role, whether at home or during the workday. P1.4 observed, "Mainly in the morning I forget ... the kids get me distracted." Similarly, P2.5 says, "I might be home, but I'm doing something, or absorbed in a task or some activity, have guests over, and it just escapes me, I forget completely."

2) Regimen complexity: Participants cited difficulties with prescriptions that were more complicated than a simple once-aday regimen. Pills taken in the middle of the day, every other day, weekly, etc. were cited by participants as more difficult to remember. Complexity was further increased by medications associated with mealtimes (taken either with an empty stomach or with food) or in conflict with other medications taken by the participant. Some participants even altered their regimens to cope with this complexity, taking extra medications or rearranging their normal medication times to fit with an altered schedule.

3) Change in routine and medications: Complex or changing schedules also contributed to missed medications. Different routines for weekends, shifting meal times, or travel/vacations all affected participants' ability to keep to a medication schedule. For example, P3.2 remarked, "I forget to take meds like right now. I sleep a little longer because I am on vacation now. I forget the meds in the morning completely." Changes in the medication regimen itself, such as a new or temporary prescription, an adjustment to dosage, or a change made in the pill's shape or color would also present difficulties, as these changes would take time to become habituated. P2.6 related this story: "Sometimes the pills change shape and color. There was a short period of time I was taking a pill that I thought was for diabetes, and my back stopped bothering me. It ended up that I was taking a pain medication."

4) Challenges in the logistics of taking medications: The logistics of medications, such as remembering to refill prescriptions and pillboxes, also presented a challenge as a diligence task for participants. In addition, participants sometimes found it difficult to take medications with them when they needed to be taken away from home, such as in the middle of the day at work or during a meal at a restaurant. P3.3 recalled "I am supposed to take a pill box in my purse, but a lot of times, when I am not the one who's driving, I don't take my purse which means my meds are at home." While many 
participants had pillboxes, there is still diligence required in filling them. P4.1 states: "so I tried the little boxes with Monday Tuesday Wednesday... and it works sometimes, but you have to sit down to fill up the pills to be taken."

\section{B. Current practices for managing medications}

Given the challenges above, participants cited a number of "domestic" techniques that they use within their living context. While a number of participants use their daily routines or friends/family to remember medications, some participants employed technological solutions to cope with medication management. They also showed their autonomy in managing medications by exerting control over regimens or emphasizing their responsibility to maintain proper medication adherence.

1) Situating medications within routines: A number of participants cited an association with some routine or event as the primary means for remembering certain medications, mentioning "patterns", "habit", "routines", or doing things "religiously." For example, a few participants mentioned keeping their pills near their bedside, or near where they have their morning coffee. Times of day seemed to have significant effect as well; a few participants mentioned either mornings or evenings as the best times for remembering their medications either due to stability ("Every pill I take is in the morning, because who knows where I am going to be in the afternoon, and sometimes life changes in the evening. No life change in the morning. [P4.2]") or by associating medication with the ritual of going to sleep or waking up or having meals ("Because of the fact that it [medication] is tied in with my meals, two of my three meals at any rate, that's sort of a warning device. I know through habit. [P5.4]").

2) Other reminders - family, one's own body: Some participants used family members or close friends as memory aids for reminders, getting text messages from them or having them bring pills along when leaving the house. In addition, the onset of symptoms, or participants' wariness of their potential onset, also served as a strong cue to remember medications, because in some circumstances, forgetting them would be potentially life-threatening. According to P3.5, "If I don't take them first thing in the morning, it is a really bad thing, so I just know immediately when I wake up."

3) Exerting control over the regimen: Interestingly, participants found ways to gain control over regimens that somehow proved difficult to remember or disruptive to their daily lives. Participants stated that in some cases they had altered their regimens to cope with this complexity, taking extra medications or rearranging their normal medication times to fit with an altered schedule. P3.2 would double-dose her medication in the morning on the expectation that she would forget her midday dose: "Sometimes, I double it up in the morning, so that I get that afternoon dose in." Another would adjust the dosage until he felt comfortable with the reduction in side effects, then report that to his doctor: "At first when I started taking some of these, you had to play with the dosage...medicine isn't an exact science... so what I did on my own, if I had a reaction to something, I'd cut it in half, and I'd tell my doctor....she'd say "Alright, see how you feel'." Supplements, perceived by most of our participants as noncritical, were taken by some on an "as-needed" basis. P1.4 states: "I take them [supplements] as I need them... I decide if I want to take them or not... Sometimes yeah [I skip supplements when] I don't feel I need it.”

4) Autonomous medication management: In many cases, participants expressed their autonomy in managing medications. Most participants emphasized that they as patients are responsible for having basic knowledge about the medications they take. In fact, most participants reported their awareness of the dosage instruction and purpose of their medications by heart. A quote from P2.4 is representative of many of our participants: "Don't need to tell me which ones to take, if I don't know which ones to take by now I'm in trouble. We all take pills every day.. we have to know which ones we take." In addition, a few participants reported doing their own research to find out about medication warnings, recalls, and generic alternatives that would help them meet goals such as saving money or feeling more comfortable with the quality of their prescriptions. For example, P2.2 remarked "whenever I get a new medication I always research it online...I have a hard time with side effects of medications so I also research that and see what it's going to do to me. Sometimes the side effects are worse than what I've got." In addition, P3.5 who takes 18 medications on a regular basis shared her own research method: "I am actually registered to I-guard. I think its .com or .org. It is a website from doctors. Every time you go in there, you list your new medicines, and they send you emails about contra indications either with the other medicines or food. Or new warnings."

\section{Perceived value of medication reminder system concept}

Participants saw value in the ability to provide multiple ways of receiving reminders, which they felt would increase the possibility of proper medication intake. For example, P1.1 said "It would be useful - I like the fact that it's also a backup. I take medication and supplements at various times of the day - it would be useful." Most participants felt comfortable being reminded on two or three different devices, preferring the mobile phone, then the TV, PC, and other devices. For example, P1.4 said "It sounds like it's a secondary backup.... I got two ways of getting the message - I have the cell phone with me most of the day but I don't have it sometimes. I like the idea of getting the secondary message."

In addition, participants expected all reminders to be linked such that one response would dismiss the reminder on all devices. Participants generally felt that concurrent reminders (multiple reminders at the same time) would be useful, but many wanted the option to configure the order of alerts to suit their own needs and/or contexts. Below, we describe how 
participants in our focus groups felt about each proposed component of our medication reminder system.

1) Perceived value of $T V$ : Most participants did not see the value of TV as a medium for "just-in-time" medication reminders. Participants cited two main limitations in terms of privacy and availability.

a) TV as a semi-public display: First, participants indicated that TV is not a private medium, as it is being shared with other guests or members of the household. This property limits the use of TV for the display of medication reminders that most participants considered private information. For example, P1.1 said, "If you have kids living with you in the house, 'daddy is on 5 medications, oh my god!'. Do you want that information to be on there? Then they start asking questions." Participants were also concerned about private information being displayed to guests visiting their home. P1.4 remarked: "I think it's a little intrusive to show the drugs [on TV]. If somebody else was in the house ... I don't think they should even know I am on 5 meds. It opens up questions 'what are you taking?' I don't want to answer any of that... It [medication] is a private matter, and when it is on the TV, it's not private."

b) Availability to watch $T V$ : Secondly, participants cited the lack of availability as another limitation of TV. Participants noted that they do not watch TV all the time, meaning that there is potential to skip a dose if they rely on their TV for reminders. For example, P3.3 remarked "because I am a stay-at-home mom, and I am not watching TV all day. I watch at a certain times of day.. I am running in and out, go back and forth with my kids, so on the TV, for my benefit, that would not help." Some participants highlighted that their TV viewing schedule does not mesh with their medication schedule, so they expressed fears of skipping medications as well. For example P1.3 cited: "Especially in the morning... You flip on the TV and you're trying to shave and shower....Especially I got to take a lot of pills in the morning so I don't know how helpful it [TV] would be for me." Also, some participants pointed out that their TV viewing schedules are different on weekends, although their medication schedule stays the same. P1.4 said "[Although I watch too much TV during weekdays] I don't watch on Saturday - I don't get up at 6 in the morning .... So I would miss my Saturday and Sunday dose."

Despite its limitations for "just-in-time" reminders, TV was seen as a possible medium to deliver secondary reminders as a backup to reminders received on a mobile phone. P1.4 said "It sounds like it's a secondary backup to TV plus the mobile. I got two ways of getting the message - I have the cell phone with me most of the day but I don't have it sometimes. I like the idea of getting the secondary message." P3.5, who takes about 18 medications on a regular basis, considered TV to be a good medium for checking the medication history. She observed, "As soon as you turn on the TV, in the morning or evening, that [reminder] comes up there...I think that would be good. Because no matter when you turn it on, when you get home, it will remind you... and you are able to do that kind of checking things at the end of the day or in the evening...oh, I forgot the third one, and it is not too late to take it. I think TV when you turn it on is a good checking point."

2) Perceived value of mobile phones: Most participants perceived mobile phones to be the most useful medium through which to receive medication reminders because mobile phones were found to be more available and private compared to TV. For example, P4.5 remarked "I like it, because I always have my phone with me, [even though] I don't always have my pills with me." P1.3 also explained the advantage of the mobile phone in terms of privacy: "It would be more discreet if it went to your mobile phone rather than your TV. Nobody else is going to look at your phone - ideal situation - less embarrassment for the people who don't like it."

3) Perceived value of home accessories: Most participants also thought that in-home accessories would be a useful medium for delivering backup reminders. Participants often cited the bedroom, bathroom, and kitchen as useful locations for receiving reminders for two reasons. First, participants usually keep their medications in those places. ("Bathroom and the kitchen are the places where I begin the medicating [P4.1]." Secondly, they perceived those places to be safe and private ("Only place that I know it will be safe is on the table in my bed where my alarm clock is. That is only place my kids don't touch [P3.3]"). In contrast, the living room seemed to be the last place to receive reminders because it was much more difficult to protect their medication information from other family members or visitors. For example, P1.5 remarked "In the bedroom, right next to the bed on the coffee table - that would be ideal. When I am outside the bedroom, the door is closed and I am in the living room with guests watching TV, they don't know about my private information and meds... Kitchen is okay - second thing. Last choice is living room."

A number of participants considered portable devices useful because it allows them determine where the reminders should be delivered. For example, P4.4 said "It would be nice if it was portable where you could move it where you want it - I'd put it right on my kitchen where I sit, where my med bottles are." In another example, P1.5 explained the usefulness of the portable device in terms of privacy concerns: "It's a good idea - you can move it into different rooms - when guests come they don't have to see it."

Other participants explained the advantages of portable devices for when they are traveling. For example, P1.1 remarked in favor of digital picture frames: "Picture frame could come in useful when you travel. If you aren't home watching $\mathrm{TV}$, on vacation for two weeks, what's going to remind you? I take pictures with me when I travel..." P4.1 also stated "It will be nice to have something you can take with you into the hotel room." While the portable alarm clock and digital picture frame were deliberately mentioned by the 
moderator as potential accessories, some other interesting ideas were discussed. For instance, some participants imagined a refrigerator magnet-type device that can be stuck to appliances or mirrors in the bathroom.

4) Perceived value of notification funtionality: When asked about a feature that would let users elect someone to be notified in case they did not take their medications properly, participants responded from two different perspectives: that of the patient, and that of a caregiver. When participants viewed themselves as patients being monitored, their overall reaction to the utility of notification was mostly negative. All participants overwhelmingly thought the notification functionality was intrusive ("invasion of privacy"), impractical ("doctors have no time to monitor my medication intake.", or costly ("It will probably be more money."). Participants, however, thought that notification of others would be useful for older or more forgetful individuals or when they put themselves in the role of a caregiver. As caregivers, the potential value of the notification function was higher, primarily for peace of mind. For example, P4.1 who was concerned about her mother living alone, remarked "My mother wanted to live alone, but I didn't feel secure in that. I would have liked to have the option of this, of programming something for her, so that I could monitor her from a distance and make sure. If she missed it three times in a row, I would be able to pop over or give her a call. I could see it as a tool for a caretaker."

\section{Experience design considerations for a medication reminder system}

During the focus groups, a number of discussions centered around the design of medication reminders in terms of their setup and delivery. We present a summary of participants' comments, and provide a set of design considerations for the development of a medication reminder system.

1) Trigger-Abstract image with audio tone: Participants across all groups expressed that they would need a simple reminder on the TV using a pictorial image and audio tone. They emphasized that getting a simple "trigger" is sufficient for them to be reminded to take their medications since they usually know the details of their regimens. Because of privacy concern, participants tended to prefer an image over a text reminder (e.g, "time to take medication"). In addition, they favored an anonymous image (such as envelope or userselectable image like a bell or cartoon) over any image that associate with medication taking such as the RX image or an actual picture of the medication. For instance, P1.3 explained: "If they use some visual reminder on TV ... rather than say 'take your medicine' maybe it can just like on my DVR if I want a reminder for an upcoming show, all it has is a picture of a bell. If it shows you a picture of a bell, reminder - take your medicine. That way it's not intrusive."

Participants expressed a preference for reminders that are somewhat intrusive to catch their attention, such as an auditory tone or flashing, because they are often not in front of the TV even when it is turned on. For example, P3.2 said "I would want some type of alarm too. Because I am not focused on the TV at the moment like when I go up the bathroom or something. Some types of beeping.. when I am outside to the yard (will be useful)."

2) Detailed information available somewhere: Despite the privacy concerns, several participants preferred having detailed medication information (e.g., the names of medications, dosage information, and specific warnings) available on the TV but in hidden places. P1.3 shared his idea of having that information in a separate (personal) place. "[I want] specific warnings like...'remember no dairy products with this' or 'hope you haven't eaten within the last hour'. It's probably hard to put it on the screen like that - maybe you can flip to a specific channel and it can give you all the warnings... like go to channel 225 or something ... maybe that's a stretch." P1.2 remarked "If you could click on the symbol somewhere else in the house and it opens up into a different screen that tells you need to take these medicines. But not the first thing that has to appear on the screen. You can open that symbol wherever you want to do it ...Y You have the option."

3) Preferences for the behavior of reminders: Most Participants generally agreed with that reminders should stay on until users dismiss them to ensure that they are seen. When no response is made, participants expect some extra action to be taken such as a) the symbol getting larger, b) the symbol changing positions, c) flashing, and d) sending a voicemail or calling the user. A snooze feature was a commonly-cited option, as it is a common feature for most alarms (e.g., cell phone alarms). For example, P4.2 said "If...you are in the middle of this particular show....and you want to get rid of it [a list of reminders], I think 'snooze all' would be kind of cool and then when the commercial comes it could come back up and you could go and do it." However, it should be noted that a wide range of preferences were discussed in terms of the behavior (e.g., how long should it stay up? Or should it disappear after $\mathrm{x}$ amount of time?) and visual presentation of reminders (e.g., size, color, selection of symbol or image, and audio)."

\section{DISCUSSION}

In this section, we summarize our findings and discuss implications for the future development of a medication reminder system and home-centered health care technology in general. First, we found that participants encountered problems through the intake and the logistics of medications. Temporary forgetfulness caused by fatigue or distraction, complexity of regimen, changes in routine and medications were the most cited challenges in taking medications. Participants also reported problems in managing the logistics of refilling and carrying medications in addition to difficulties in reconciling regimens with their own lifestyles or physical conditions. In 
response to these challenges, participants reported various "domestic techniques" to remember medications such as the use of daily routines (e.g., taking medicines in the morning near where they have a morning coffee), the use of family members as a memory aid (e.g., wife sending text messages), and the use of artifacts (e.g., pill boxes and wearable alarms).

As described in the related work section, previous research identified similar challenges and practices, but they mainly focused on the elderly patients. For example, Palen and Aaløkke [10] studied with elders living within the Danish healthcare system who received daily visits from healthcare workers and found that they devise their own medication management practices using the spatial and temporal rhythms of their days to aid remembering. In the present study, we also identified similar practices from different demographics: middle-aged and elderly living independently in their own homes. We claim, therefore, that several of the distributed cognitive behaviors outlined in [10] can be extended to these demographics as well.

However, we identified a number of additional practices and attitudes from our participants which we believe stem from increased autonomy and independency compared to the elders in [10]. By focusing on their autonomy, our work brings additional implications regarding the use of in-home healthcare technology. We discuss some of these implications below.

\section{A. Needs for customizable or context-aware reminders}

While participants agreed with the benefits of receiving medication reminders, they were not in favor of rigid reminders that push alerts based on a fixed schedule. They often insisted that they usually take medications on schedule; therefore, receiving reminders for every medication as well as responding to the reminders would be more disruptive to their lives than helpful. They reported that their potential use of a reminder system would be limited to certain medications of interest, including 1) medications that they forget in the morning or in the evening, 2) medications with a complex regimen (e.g., one that is taken every other day or throughout the day), 3) new medications that haven't yet been incorporated into their daily routines. This implies that as a vast range of variation exists in their routine and medication schedule, medication reminders need to be customizable to their own needs. Designers should avoid defining prescriptive models of use while offering a wide range of customization options for the reminder settings [10].

We also found that potential obstacles for continued usage of reminder systems are the perceived effort ("too much work") and the novelty factor ("After a while you're going to become numb to it"). Again, most participants reported that they are likely to take most medications on time without prompting. Not only does the alert now lose its value as a reminder, but it potentially places an added burden on the user to acknowledge it (e.g., to indicate compliance or prevent its recurrence). At the same time, participants did indicate interest in using responses as a tracking mechanism for personal use (e.g. to recall if/when they took medications) as well as for review with their doctors.
This indicates a need for smart alert technologies that will (a) trigger visible/audible alerts only when it is deemed necessary, thereby reducing the cognitive overload on users and (b) automate responses for tracking purposes. Automated response can be driven by inferences based on observed user actions and other sensory input. For instance, networked pillboxes [15] could be used to detect compliance events (e.g., user took his medication) and suppress upcoming alerts or register responses to recent alerts in an automated manner that will reduce user effort but continue to support tracking requirements.

Also, this may indicate a need for persuasive technologies that can sustain the user's interest and adoption of such systems after the initial novelty wears off. Current research in using context to mitigate the perceived 'interruption' burden of alerts [16] and adopting different strategies for long-term vs. shortterm adherence [17] may be particularly relevant in designing such interruption-driven notification systems.

\section{B. Needs of information search}

We found that users had strong needs for technology that provides information on their current medications. This information was seen as supporting their continued autonomy in managing medications. Some participants reported doing their own research on drug interactions, side effects, and availability of generics while altering their medication intake on their own to cope with particularly complex or burdensome regimens. To support these activities, designers may want to consider ways of integrating this independent research with users' existing healthcare networks. In this fashion, people can continue to conduct their research, while their healthcare providers (doctors or pharmacists) can offer suggestions or clarifications as needed.

We also found that participants perceived the pharmacy to be a more trusted resource on medications than their doctors. Therefore, they wanted to have more advanced services from the pharmacy, such as automatic prescription refill and delivery, and updates on drug interactions, side effects, generic drug options, and recalls or lawsuits. It seems that developing technologies and services that enable pharmacy care programs would be the most promising opportunity. Previous empirical research [18] found in a randomized controlled trial that a comprehensive pharmacy care program which provided patients with individualized education on medication (i.e., teaching drug names, indications, strengths, adverse effects and usage instructions) led to increases in medication adherence, medication persistence, and clinically meaningful reductions in blood pressure. The results of our study suggest that the TV will be more suitable for delivering educational materials or informative notifications from a pharmacy rather than displaying medication reminders.

\section{Value of portability in medication reminders}

We found that mobile phones would be the most useful medium for delivering medication reminders because of their constant accessibility and privacy compared to a semi-public display such as the TV. This may explain why a mobile phone 
has been a popular platform for medication reminder or adherence systems in both academic and industrial settings $[8,9,19,20]$. However, mobile phones, when they are used alone for delivering reminders in the home, may present challenges, as mobile phones are not always kept close at hand within the home [21]. The result of our study suggests that concurrent multiple reminders on both a mobile phone and inhome accessories would lead to better results in medication adherence than a single reminder on the mobile phone.

Portability is seen as an important attribute of any such reminder system because it allows flexibility in reminder placement. As O'Brien et al. [22] highlighted in their ethnographic study, interaction with technology in the home is a managed activity that is closely linked to relationships with others in the home. We also found in this study that there is varying ownership of space within the home at certain times. Certain spaces within the home clearly "belong" to particular members of the household. For example, a participant in our study who was a stay-at-home mom clearly indicated that the bedroom is the only place that "belongs" to her. Since the specifics of which spaces belong to whom differ from household to household, we argue that portable in-home accessories such as digital picture frames allow users to best manage their privacy from other family members or visitors.

This study confirmed the notion that most participants see their medication management as a very private matter [23]. The TV, usually the centerpiece of home technology, turned out to be problematic when used to display medication reminders due to its very public nature. Abstract reminders (such as an image of a bell or a tone) may make the use of TV acceptable, but any further details should be restricted to more private displays. We also found a tendency for the middle aged groups to be more sensitive to the privacy issue than the elderly groups, although it can vary depending on other factors such as their health status and social connectedness. This suggests that designers of home centered health care systems need to understand how users' managed activity links with other family members and address the privacy issues up-front before the system development

\section{ACKNOWLEDGEMENTS}

The authors acknowledge the contribution of anonymous reviewers, some of whose comments and suggestions are incorporated into this article. The authors would also like to express their gratitude to Crysta Metcalf and Elaine Huang for their feedback to improve the study design.

\section{REFERENCES}

[1] M.C. Sokol, K.A. McQuigan, R.R. Verbrugge, and R.S. Epstein. "Impact of medication adherence on hospitalization risk and healthcare cost," Med Care 2005, vol. 43, pp. 521-530.
[2] "American Heart Association, Statistics You need To Know," available online at http://www.americanheart.org/presenter.jhtml?identifier=107

[3] "Enhancing Prescription Medicine Adherence: A National Action Plan," A report by the National Council on Patient Information and Education (NCPIE), 2007.

[4] H.P. McDonald, A.X. Garg, and R.B. Haynes. "Interventions to enhance patient adherence to medication prescriptions: Scientific review," JAMA 2002, vol. 288, pp.2868-2879.

[5] A. Atreja, N. Bellam, and S.R. Levy. "Strategies to enhance patient adherence: Making it simple," Medscape General Medicine 2005, vol. 7, No. 2.

[6] R.L. Ownby, "Medication adherence and cognition: Medical, personal and economic factors influence level of adherence in older adults," Geriatrics 2006, vol. 61, no. 2, pp.30-35.

[7] L. Osterberg and T. Blaschke "Adherence to medication," New England Journal of Medicine 2005, vol. 353, pp. 487-497.

[8] P. Kaushik, S.S. Intille, and K. Larsen, "User-adaptive reminders for home-based medical tasks. A case study," Methods of Information in Medicine 2008, vol. 47, pp. 203-207.

[9] K. Fishkin and M. Wang, "A Flexible, Low-Overhead Ubiquitous System for Medication Monitoring," Intel Research Technical Report IRS-TR-03-011, Oct 2003.

[10] L. Palen and S. Aaløkke, "Of pill boxes and piano benches: "homemade" methods for managing medication", Proceedings of the 2006 20th anniversary conference on Computer supported cooperative work, November 04-08, 2006.

[11] S.A. Ballegaard, J.Bunde-Pedersen, and J.E. Bardram, "Where to, Roberta?: reflecting on the role of technology in assisted living," NordiCHI 2006, October 14-18, pp.373-376.

[12] G.J. Thompson, W.B. Locander, and H.R. Pollio, "Putting consumer experience back into consumer research: The philosophy and method of existential-phenomenology," Journal of consumer research 1989, vol. 16 , no. 2, pp.133-146.

[13] A. Strauss and J. Corbin, "Basics of qualitative research: Grounded theory procedures and techniques," London, UK: Sage, 1990.

[14] MAXQDA: http://www.maxqda.com/

[15] MedSignals Digital Pillbox https://www.medsignals.com/default.aspx

[16] J. Ho and S.S. Intille, "Using context-aware computing to reduce the perceived burden of interruptions from mobile devices," Proceedings of the SIGCHI 2005, Portland OR, pp. 909-918.

[17] T. Bickmore, D. Mauer, F. Crespo, and T. Brown, "Persuasion, task interruption and health regimen adherence," Proc. Persuasive Technology 2007, LNCS 4744, pp. 1-11.

[18] J.K. Lee, K.A. Grace KA, and A.J. Taylor, "Effect of a pharmacy care program on medication adherence and persistence, blood pressure, and low-density lipoprotein cholesterol: a randomized controlled trial," JAMA 2006, vol. 296, pp.2563-2571.

[19] The Pill Phone: https://www.pillphone.com/

[20] The On Time RX: http://www.ontimerx.com/mobile/welcome.php

[21] S.N. Patel, J.A. Kientz, G.R. Hayes, S. Bhat, and G.D. Abowd. "Farther than you may think: An empirical investigation of the proximity of users to their mobile phones," Proceedings of Ubicomp 2006, pp. 123-140.

[22] J. O'Brien, T. Rodden, M. Rouncefield, and J. Hughes, "At home with technology: An ethnographic study of a set-top box trial," ACM Transactions on HCI 1999, vol. 6, No. 3, pp. 282-308.

[23] T. Hirsch, J. Forlizzi, E. Hyder, J. Goetz, C. Kurtz, and J. Stroback, "The ELDer project: social, emotional, and environmental factors in the design of eldercare technologies," In Proceedings on Universal Usability 2000 , pp. $72-79$. 